\title{
Dental X-ray Image Segmentation using Gaussian Kernel-Based in Conditional Spatial Fuzzy C-means
}

\author{
Arna Fariza ${ }^{\# *}$, Agus Zainal Arifin", Eha Renwi Astuti ${ }^{\$}$ \\ ${ }^{\#}$ Informatics Engineering Dept., Institut Teknologi Sepuluh Nopember, Kampus ITS Sukolilo, Surabaya, 60111, Indonesia \\ E-mail:arna@pens.ac.id \\ *Informatics and Computer Engineering Dept., Politeknik Elektronika Negeri Surabaya, \\ Jl. Raya ITS - Kampus PENS Sukolilo, Surabaya 60111, Indonesia \\ E-mail:agusza@cs.its.ac.id \\ ${ }^{\$}$ Dentistry Dept. Airlangga University, Kampus B UNAIR - Jl. Airlangga 4-6, Surabaya, 6028, Indonesia \\ E-mail: eharenwi@gmail.com
}

\begin{abstract}
Dental X-ray image segmentation is a difficult task because of intensity inhomogeneities among various regions, low image quality due to noise and low contrast errors of data scanning. In this paper, we proposed a new conditional spatial fuzzy $\mathrm{C}$-means algorithm with Gaussian kernel function to facilitate dental $\mathrm{X}$-ray image segmentation. The Gaussian kernel function is used as an objective function of conditional spatial fuzzy $\mathrm{C}$-means algorithm to substitute the Euclidian distance. Performance evaluation of the proposed algorithm was carried on dental $\mathrm{X}$-ray from different teeth of some panoramic radiographs. The average of false negative fraction (FNF) and false positive fraction (TPF) values using proposed algorithm better than conditional spatial fuzzy C-means algorithm but vise versa for true positive volume fraction (FPF) value. The segmentation result of the proposed algorithm effectively recognizes tooth region as main part of the dental $\mathrm{X}$-ray image.
\end{abstract}

Keywords — dental x-ray image; fuzzy c-means; spatial information; gaussian kernel-based

\section{INTRODUCTION}

Segmentation of dental X-ray image plays an important rule to analyze dental $\mathrm{X}$-ray images in the field of practical dentistry such as diagnostic support and identification tools to obtain a valuable information [1]. Image segmentation is one of an important task in image processing and image analysis, such as pattern recognition and image matching [2].

Dental X-ray consists of three main parts: tooth area, dental structure area and background area [3] as shown in Fig. 1. The tooth area has a high grayscale value, and it is the main part of the image. Dental structure area has a medium grayscale value and consists of gum, bone and periodontitis structure. The background has the smallest grayscale value among them and shows the background of the teeth structure. Grayscale color differences among pixels were not significant between the element of tooth structure such as enamel, dentin, and pulp [4].

Performance of dental X-ray images segmentation is decreased because they have intensity inhomogeneities among various parts, a low-quality image due to noise and low contrast of data scanning [5]. Those conditions make difficulty in distinguishing the parts of teeth. Hence, robust segmentation algorithms are required to classify the area of the dental X-ray image.

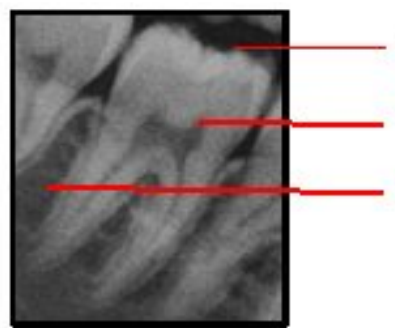

background area

tooth area

dental structure area

Fig. 1 The three main parts of dental x-ray: tooth area, dental structure area, and background area

The Fuzzy C-means (FCM) clustering method is widely used in classification problem effectively [6]. It divides an object into groups that have a higher degree of similarity than any other group. The clustering method is divided into two main categories. FCM is a soft clustering method that uses a membership matrix indicating the membership level of data points to different groups. Therefore, data points can be owned by more than one cluster. FCM is an effective soft 
clustering method $[7,8]$ that has some benefits. Unlike hard clustering methods, such as the k-means algorithm, which assign pixels exclusively to one cluster, the FCM algorithm allows pixels to have relationships with multiple clusters with varying membership levels and thus it makes more sense in real applications. Because of this flexibility, FCM has been widely used in medical image segmentation.

There are several disadvantages of the conventional FCM algorithm, such as, it does not consider spatial information from the image, and also poor initialization leads to locally optimal solutions. Therefore, this algorithm is very sensitive to noise and imaging artifacts. There has been spatial information involvement to neighboring pixels as a part of FCM optimization procedures [9], [10], [11]. Those incorporated the spatial information into the membership function of FCM. The spatial function is considered to summarize the membership value of each neighboring pixels. The spatial clustering algorithm has some advantages, included providing regions homogeneously, reducing the spurious blobs and removing the noisy spot. conditional spatial fuzzy C-means (csFCM) incorporated conditioning effects that were represented by a conditional variable, which described a level of involvement of the pixel to construct clusters and spatial information of each pixel into the membership function [11]. It had been applied to magnetic resonance imaging (MRI) data, and it was effective to reduce sensitivity to noise and intensity inhomogeneities by incorporating local and global spatial information into a weighted membership function.

The other drawback of the FCM algorithm is a number of points in the clusters that almost equal; nearly all points do not have a membership value of one cluster and the outliers data always affect a poor clustering result [12], [13]. Euclidean distance is widely used to determine the shortest distance between two pixels intensity values. However, Euclidian distance has failed to detect outlier data. There is some literature to generalize FCM is proposed to overcome the drawbacks of Euclidian distance. On the other hand, some recent machine learning methods have been used to construct a nonlinear version of a linear algorithm using kernel method, e.g., Support Vector Machine (SVM) [14], Kernel Principal Component Analysis (KPCA) [15] and Kernel Fisher Discriminant (KFD) [16]. Kernel method has been applied to unsupervised clustering [17], [18]. It substituted kernel as a nonlinear map implicitly from the low dimension data space to the higher dimensional feature space. Kernel version of FCM with spatial constraints for image segmentation overcome the lack clear of cluster result due to the use of high dimensional feature space and generate a robust cluster [19]. A Gaussian kernel-based FCM algorithm with a spatial bias correction has already presented image segmentation of noisy image [12]. Gaussian kernel function that has used in this algorithm shows an effective medical image segmentation result even if they have a far distance intensities. It can control the clustering result to be interpreted in the original data space using simpler and inexpensive computational.

Dental X-ray images that have intensity inhomogeneities of various regions, low image quality due to noise and low contrast error of data scanning require robust fuzzy segmentation algorithm by observing the global interaction and local interaction among neighboring pixels and Gaussian kernel-based adequate to handle normal distribution of dental X-ray intensity pixels. In this paper, we proposed a new conditional spatial fuzzy C-means algorithm with Gaussian kernel function to facilitate dental X-ray image segmentation. The Gaussian kernel function is used as an objective function of conditional spatial fuzzy C-means algorithm to substitute the Euclidian distance. We verified the new conditional spatial information fuzzy C-means with Gaussian kernel-based algorithm on dental X-ray ray from different teeth of some panoramic radiographs. The results of this segmentation algorithm on dental X-ray images can be used in practical dentistry for further clinical evaluation such as dental caries, periodontal disease, osteoporosis, tooth disorders and human forensic identification.

The remaining structure of this paper is organized as follow: Section II explains the background of the proposed spatial information FCM with the gaussian kernel-based algorithm. Section III explain the methodology of proposed dental x-ray segmentation algorithm. Section IV reports the experimental results and discussion. Section V concludes this paper.

\section{MATERIAL AND METHOD}

In this section, the background algorithms are first briefly reviewed. We illustrate three typical algorithms that related to our approach.

\section{A. The Fuzzy C-means Algorithm}

The fuzzy C-means (FCM) algorithm is a fuzzy clustering method developed by Bezdek represent the data cluster with minimizing the quadratic criteria[8]. The set of $n$ data $X=$ $\left\{x_{1}, x_{2}, \ldots, x_{n}\right\}$ will be partitioned by calculating the center of clusters $a$ and the membership matrix $U$ where array $U=$ $\left[\mu_{i j}\right]$. The objective function $J_{m}$ where $m$ controls fuzziness degree is defined as

$$
J_{m}(\mu, a)=\sum_{j=1}^{n} \sum_{i=1}^{c} \mu_{i j}^{m}\left\|x_{j}-a_{i}\right\|^{2}
$$

The objective function $J_{m}$ affects the membership function $\mu_{i j}$

$$
\mu_{i j}=\frac{\left\|x_{j}-a_{i}\right\|^{-\frac{2}{m-1}}}{\sum_{k=1}^{c}\left\|x_{j}-a_{k}\right\|^{-\frac{2}{m-1}}}
$$

and the cluster centers $a_{i}$ represented as (3).

$$
a_{i}=\frac{\sum_{j=1}^{n} \mu_{i j}^{m} x_{j}}{\sum_{j=1}^{n} \mu_{i j}^{m}}
$$

The cluster centers represent the local minimum of the FCM objective function. Convergence of a solution is detected by comparing the changes in the membership function or the cluster center in every iteration steps. Steps of standard FCM algorithms are

Input: Specify the number of clusters $c$, the degree of fuzziness $m=2$ and the error $\varepsilon$.

Step 1: Initialize randomly the centers of clusters $a_{i}^{(0)}$

Step 2: For $t=1,2, \ldots, t_{\max }$ do

- Calculate the membership value $U^{(t)}$ using the centers $a_{i}^{(t-1)}$ using Eq. (2) 
- Update the centers $a_{i}^{(t)}$ using $U^{(t)}$ in Eq. (3)

- if $\left\|\mathrm{a}_{\mathrm{i}}^{\mathrm{t}}-\mathrm{a}_{\mathrm{i}}^{\mathrm{t}-1}\right\|<\varepsilon$ then stop

Step 3: Return the cluster center $a_{i}$ and the membership value $\mu_{i j}$; where $i=1,2, \ldots, c$; and $k=1,2, \ldots, n$.

\section{B. The Conditional Spatial Fuzzy C-means Clustering Algorithm}

Conventional FCM technique does not consider the spatial information of neighboring pixels, thus resulting from incorrect classification because of an abnormal feature image. One of the important characteristics of the image is that neighboring pixels have a high correlation among them. In other words, the neighboring pixels have similar feature values and high probability of same cluster belonging.

Conditional spatial fuzzy C-means (csFCM) incorporated local spatial interaction among neighboring pixels in the fuzzy membership function [10], [11]. If the neighboring pixel shares similar characteristics, the center pixel should have a higher probability of grouping to the same cluster as the neighboring pixel. The conditional variable is used to describe the level of involvement as a weighted membership function. The spatial information is represented using a spatial function $h_{i j}$.

$$
h_{i j}=\frac{\sum_{k \in N B\left(x_{j}\right)} \mu_{i k}}{M}
$$

Notation $h_{i j}$ is the probability that pixels $x_{j}$ belongs to the $i$ th cluster. $N B\left(x_{j}\right)$ represent a square window of pixel $x_{j}$ in the spatial domain (for example $3 \times 3,5 \times 5$ or $7 \times 7$ ) and $M$ is the number of neighboring pixels. The spatial function $h_{i j}$ represent that a cluster is large if the majority of its neighborhood belongs to the same cluster. The membership of spatial information function $u_{i j}$ represents the probability of the pixel is derived from the standard FCM membership function as

$$
u_{i j}=\frac{h_{i j}\left\|x_{j}-a_{i}\right\|^{-\frac{2}{m-1}}}{\sum_{k=1}^{c}\left\|x_{j}-a_{k}\right\|^{-\frac{2}{m-1}}}
$$

The global membership value $\mu_{i j}$ and local membership value $u_{i j}$ are independently each other. So, they are combined into weighted membership function $z_{i j}$.

$$
z_{i j}=\frac{\left(\mu_{i j}\right)^{r}\left(u_{i j}\right)^{s}}{\sum_{k=1}^{c}\left(\mu_{k j}\right)^{r}\left(u_{k j}\right)^{s}}
$$

Respectively, parameter $r$ and $s$ control the importance level of both the membership functions and spatial membership functions. The $z_{\mathrm{ij}}$ equation can reduce the weight of clusters in noisy pixels with information value from neighboring pixels, and it will reduce noisy area or spurious blobs. But in a homogeneous region, the spatial function only reinforces the original membership and resulting from an unchanged clustering. Conditional spatial FCM with parameters $r$ and $s$ is defined as $\operatorname{csFCM}_{r, s}$. $\mathrm{csFCM}_{l, 0}$ means the conventional FCM. The weighted membership function causes the updated cluster centers $w_{i}$.

$$
w_{i}=\frac{\sum_{j=1}^{m} z_{i j}^{m} x_{j}}{\sum_{j=1}^{m} z_{i j}^{m}}
$$

\section{Gaussian Kernel-induced FCM}

Several kernel-based learning machines, e.g., Support Vector Machine (SVM) [14], Kernel Principal Component Analysis (KPCA) [15] and Kernel Fisher Discriminant (KFD)
[16] have been found for pattern recognition applications. These algorithms were based on the kernelized process with an implicit nonlinear map, from the data space $\left\{x_{1}, \ldots, x_{n}\right\} \subseteq$ $X$ mapped to feature space, $\Phi: X \rightarrow F(x \rightarrow \Phi(x))$. A nonlinear original input data set which has low dimension data space is mapped into a higher-dimensional feature space by inner product function $F$ as linear ones.

A kernel in the feature space can be represented as a function $K$ as

$$
K(x, y)=\langle\Phi(x), \Phi(y)\rangle
$$

$\langle\Phi(x), \Phi(y)\rangle$ represent the inner product operation. The interesting point of kernel function is that the inner product $F$ can be implicitly computed without explicitly knowing the nonlinear mapping of feature space $\Phi$. Therefore, kernels allow inner products computation in feature spaces.

The objective function of kernelized FCM algorithm represents the clustering center as the linear combining of all $\Phi\left(x_{j}\right)$ from Eq. (1), thus the objective function which lies in feature space is defined as

$$
J_{m}(\mu, a)=\sum_{j=1}^{n} \sum_{i=1}^{c} \mu_{i j}^{m}\left\|\Phi\left(x_{j}\right)-\Phi\left(a_{i}\right)\right\|^{2}
$$

Feature space $\Phi$ is an implicit nonlinear map, thus feature center $\Phi\left(a_{i}\right)$ is not expressed as a linear combining sum of all feature data $\Phi\left(x_{j}\right)$, but it still considered as a mapped point (image) of $a_{i}$ in the original data space. Thus, the kernel substitution can be derived as

$$
\begin{aligned}
& \begin{array}{l}
\left\|\Phi\left(x_{j}\right)-\Phi\left(a_{i}\right)\right\|^{2} \\
=\left(\Phi\left(x_{j}\right)-\Phi\left(a_{i}\right)\right)^{T}-\left(\Phi\left(x_{j}\right)-\Phi\left(a_{i}\right)\right) \\
=\Phi\left(x_{j}\right)^{T} \Phi\left(x_{j}\right)-\Phi\left(a_{i}\right)^{T} \Phi\left(x_{j}\right)-\Phi\left(x_{j}\right)^{T} \Phi\left(a_{i}\right) \\
\quad+\Phi\left(a_{i}\right)^{T} \Phi\left(a_{i}\right) \\
=K\left(x_{j}, x_{j}\right)+K\left(a_{i}, a_{i}\right)-2 K\left(x_{j}, a_{i}\right)
\end{array}
\end{aligned}
$$

Gaussian radial basis function (RBF) is used for kernel function definition where $K\left(x_{j}, x_{j}\right)=1$, then (10) can be simplified to $1-K\left(x_{j}, a_{i}\right)$ [12]. The equation of $K\left(x_{j}, a_{i}\right)$ is defined as

$$
K\left(x_{j}, a_{i}\right)=\exp \left(\frac{-\left(\sum_{i=1}^{d}\left|x_{i}-a_{i}\right|^{r}\right)^{s}}{\sigma^{2}}\right)
$$

Notation $d$ is the dimension of vector $x$ and $r \geq 0$ and $1 \leq s$ $\leq 2$. Notation $\sigma^{2}$ is a variance of the image intensity. Because of variance $\sigma$ is presented as a dispersion, we use sample variance to estimate $\sigma^{2}$ with

$$
\sigma^{2}=\frac{\sum_{i=1}^{d}\left\|x_{i}-\bar{x}\right\|^{2}}{n} \text { and } \bar{x}=\frac{\sum_{i=1}^{d} x_{i}}{n}
$$

If the kernel function is incorporated into radial basis function $(r=2, s=1)$, the equation can be simplified as

$$
1-K\left(x_{j}, a_{i}\right)=1-\exp \left(-\frac{\left\|x_{j}-a_{i}\right\|}{\sigma^{2}}\right)
$$




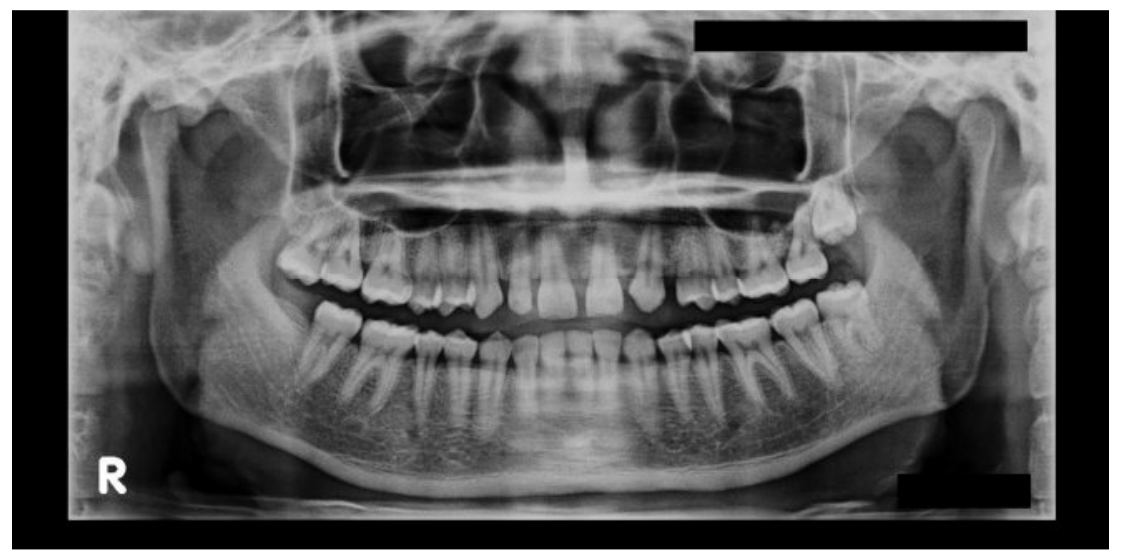

(a)

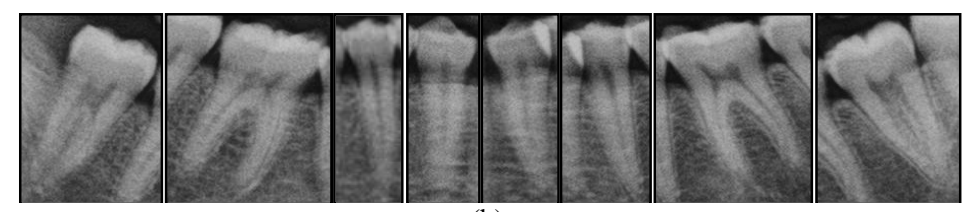

(b)

Fig. 1 (a) Panoramic image; (b) part of the mandibular teeth: right molar, right premolar, left premolar and left molar The three main parts of dental x-ray: tooth area, dental structure area, and background area

An exponential distance from Eq. (13) causes the coefficient of kernel function ranging from zero to one. It interprets the feature space into the data space using simpler and inexpensive computational. The Gaussian kernel-based efficiently classify the pixel. The radial basis function that used in the kernelized process causes the coefficient in the range of zero to one, and it is consistent with the FCM rules.

Then, the objective function is derived from (9) according to Gaussian kernel-based function as

$$
J S_{m}^{k}(\mu, a)=\sum_{i=1}^{c} \sum_{j=1}^{n} \mu_{i j}^{m}\left(1-K\left(x_{j}, a_{i}\right)\right)
$$

This objective function eq. (14) affect the membership function $\mu_{i j}$ and the membership function of the spatial information $u_{i j}$ of the conditional spatial fuzzy c-means (csFCM). This kernel function is robust to inhomogeneities, noise, and outliers when it was decreasing.

\section{Methodology}

In this section, we illustrate steps for dental X-ray image segmentation. Clustering is performed by assigning labels to each pixel: the "main sections" (areas of the tooth) or "background sections" (areas of background and tooth structure).

The data inputs are collected from the clinic of Faculty of Dentistry, Airlangga University, Surabaya, Indonesia, in panoramic image form. The original dental X-ray data is divided into some part of the teeth as shown in Fig. 2. Fig. 2(a) is the panoramic image, and observed images are the mandibular areas. The observed panoramic image is divided into some part of teeth as shown in Fig. 2(b). The mandibular teeth are manually disparted into right molar 2, right molar 1 , right premolar 2 , right premolar 1 , left premolar 1, left premolar 2, left molar 1 and left molar 2.

The input of dental $\mathrm{x}$-ray image in Fig. 2(b) is processed to determine the background region and tooth region as the main part. The flowchart of the dental x-ray segmentation process is shown in Fig. 3.

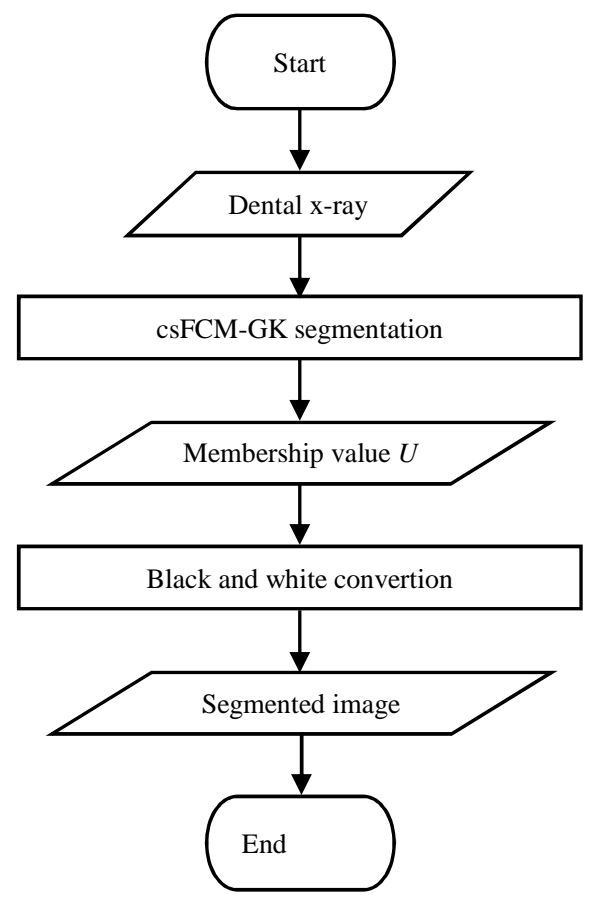

Fig. 3 Dental x-ray image segmentation process framework

\section{E. The Proposed Conditional Spatial FCM with Gaussian Kernel-based}

Conditional spatial FCM (csFCM) has superior segmentation result of MRI image by combining global and local information into weighted membership function. However, dental X-rays image that has inhomogeneities and low quality required a robust segmentation algorithm. Gaussian distribution can properly handle the random 
distribution data such as dental X-ray image. Therefore, the Gaussian kernel-based is appropriate for dental x-ray images that have normal distribution data.

The original dental X-ray as shown in Fig. 4(a) is processed using conditional spatial fuzzy C-means with a Gaussian kernel (csFCM-GK) and divided into two clusters that indicate the background and the tooth part.

The objective function $J S_{m}^{k}$ in (14) derives the membership function $\mu_{i j}$ (15) and cluster center $a_{i}$ (16).

$$
\mu_{i j}=\frac{\left(1-K\left(x_{j}, a_{i}\right)\right)^{-\frac{2}{m-1}}}{\sum_{k=1}^{c}\left(1-K\left(x_{j}, a_{k}\right)\right)^{-\frac{2}{m-1}}}
$$

and

$$
a_{i}=\frac{\sum_{j=1}^{m} \mu_{i j}^{m} K\left(x_{j}, a_{i}\right) x_{j}}{\sum_{j=1}^{m} \mu_{i j}^{m} K\left(x_{j}, a_{i}\right)}
$$

Gaussian RBF also affects the membership of spatial information function $u_{i j}$ that represents the probability of the neighboring pixel. A new membership of spatial information function is derived from (5) as

$$
u_{i j}=\frac{h_{i j}\left(1-K\left(x_{j}, a_{i}\right)\right)^{-\frac{2}{m-1}}}{\sum_{k=1}^{c}\left(1-K\left(x_{j}, a_{k}\right)\right)^{-\frac{2}{m-1}}}
$$

This Gaussian membership function and spatial information function get better identifying the similarity of the whole and neighboring pixels, then affect to handle the noise and outlier pixel. The proposed Gaussian RBF for conditional spatial information fuzzy C-means algorithms can be summarized in the following step

Input: Specify the values for the number of clusters $c$, the degree of fuzziness $m, r, s$ and the error $\varepsilon$.

Step 1: Initialize randomly the centers of clusters $a_{i}^{(0)}$ and the center of joint cluster $w_{i}^{(0)}$

Step 2: For $t=1,2, \ldots, t_{\max }$ do

- Calculate the membership value $U^{(t)}$ using the centers $a_{i}^{(t-1)}$ using Eq. (15)

- Calculate the conditional spatial membership value $u_{i j}$ ${ }^{(t)}$ using the centers $w_{i}^{(t-1)}$ using Eq. (17)

- Calculate weighted membership value $z_{i j}^{(t)}$ using Eq. (6)

- Update the center of joint cluster $w_{i}^{(t)}$ using Eq. (7)

- Update the centers $a_{i}^{(t)}$ using $U^{(t)}$ using Eq. (16)

- if $\left\|\mathrm{a}_{\mathrm{i}}^{\mathrm{t}}-\mathrm{a}_{\mathrm{i}}^{\mathrm{t}-1}\right\|<\varepsilon$ then stop
Step 3: Return the center of joint cluster $w_{i}$ and the weighted membership value $z_{i j} ; i=1,2, \ldots, c ; k=1,2, \ldots, n$.

The user defines the parameters of the csFCM-GK such as a number of cluster $c$, the fuzzifier $m$, stopping threshold $\mathcal{E}$, square window $N B$, a conditional parameter of spatial information $r$ and $s$. The suggested value of those parameters in the relevant articles are often $c=2$ (background and tooth part), $m=2$ and $\varepsilon=0.001$ [10], and $N B$ $=3, r=2$ and $s=2$ [11]. Nonetheless, we can adjust the parameters for specific analysis. The variance $\sigma^{2}$ is calculated from the global variance pixels and neighborhood variance pixels.

The outcomes of this process are the final centers and membership value $U$. The region with the lowest density corresponding to the background region and the highest density corresponding to the tooth region. The outputs of csFCM-GK algorithm consist of membership value $U$ of tooth region and background region as shown in Fig. 4(b) and Fig. 4(c). The membership value $U$ of tooth area and background area are in the range of zero to one. The value of the cluster center obtained from the csFCM-GK segmentation consists of two cluster center values. For example, csFCM-GK process for dental x-ray image in Fig. 4 gets the cluster center value [124.85, 66.84]. The highest value cluster indicates tooth region, and the lowest value indicates the background region.

\section{F. Black and White Conversion}

The final step of the segmentation algorithm is converted the membership value in zero and one range into black and white color to visualize the output. The membership function outputs are assumed to approximate the optimal result. Therefore $U$ is used to clarifying and changing to the binary image: black for the background and white for the tooth. The membership value that near the lowest center indicate the background and the biggest center indicate the tooth. If the lowest center is $c_{1}$ and the biggest center is $c_{2}$, each pixel is labeled based on its gray value $(f(x))$, then the binary values $g(x)$ are defined as

$$
g(x)=\left\{\begin{array}{lr}
0 & \text { if }\left(\operatorname{round}\left(f(x), c_{1}\right)=1\right. \\
255 & \text { else }
\end{array}\right.
$$

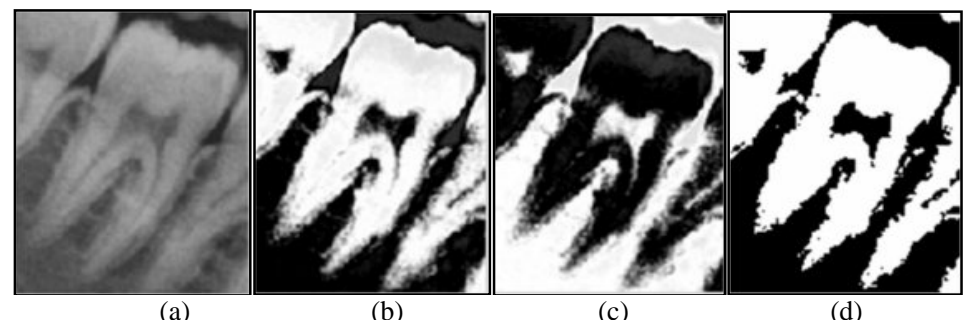

Fig. 4 Segmentation process example (a) original dental x-ray; (b) membership value $U$ of tooth region of csFCM-GK; (c) membership value $U$ of background region of csFCM-GK; (d) clustering image result in black and white color; (e) output result 


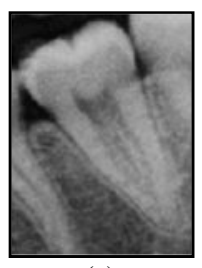

(a)

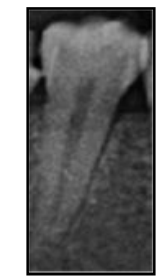

(a)

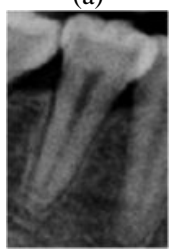

(a)

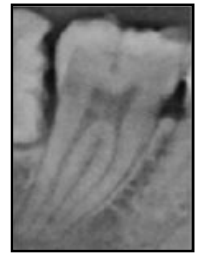

(a)

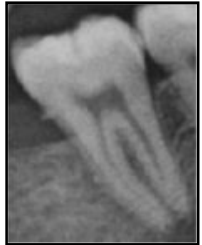

(a)

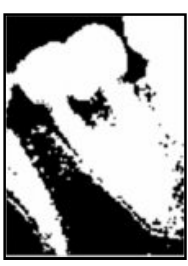

(b)

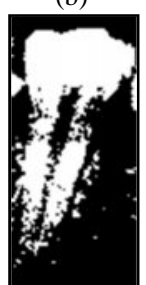

(b)

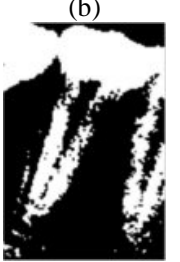

(b)

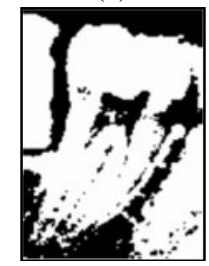

(b)

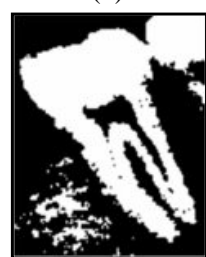

(b)

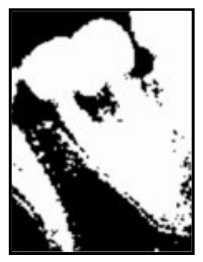

(c)

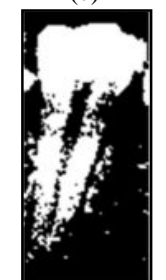

(c)

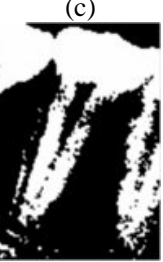

(c)

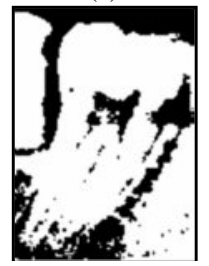

(c)

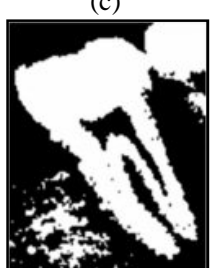

(c)

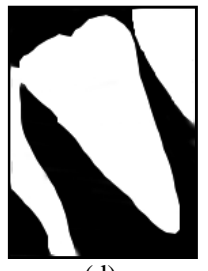

(d)

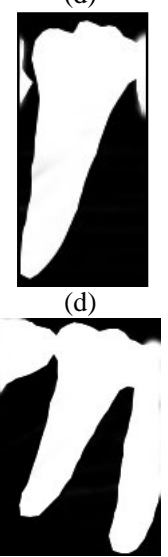

(d)

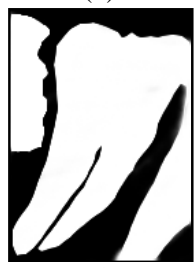

(d)

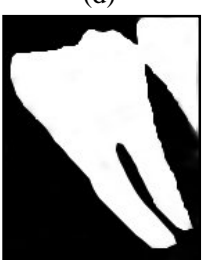

(d)

Fig. 5 Comparison of segmentation result (a) original image; (b) segmentation results of csFCM; (c) segmentation results of csFCM-GK; (d) ground truth

\section{RESULT AND DISCUSSION}

\section{A. Evaluation Criteria}

In this section, we perform an experiment using the evaluation criteria used in [20] literature to measure the accuracy of the proposed segmentation algorithm. The evaluation compares the segmentation area between depicted area by the doctor manually (that called ground truth) and resulted area by the proposed segmentation algorithm.

Accuracy evaluation of the proposed segmentation algorithm consists of three indexes, false negative fraction $(F N F)$, false positive fraction $(F P F)$ and true positive volume fraction $(T P F)$ [20]. The correlation of those indexes is illustrated in Fig. 4. TPF index is the intersection of $F N F$ and FPF index. FNF defines the total 'true' fraction region that is failed to be segmented by the segmentation method. FPF defines the number of background regions that contained within the segmentation area, and TPF states the total 'true' region contained within the segmentation area. Therefore, a smaller $F N F$, a smaller $F P F$, and a larger $T P F$ indicate better segmentation performances. These three indexes are credible to evaluate dental X-ray image segmentation.

Suppose the segmentation area specified by the doctor is denoted by $T$, and the segmentation area of the proposed algorithm is denoted by $D$, then the criterion is defined as

$$
\begin{gathered}
F N F=\frac{T \cup D-D}{T} \\
F P F=\frac{T \cup D-T}{T} \\
T P F=\frac{T \cap D}{T}
\end{gathered}
$$

\section{B. Experiment Result}

The proposed segmentation method csFCM-GK is tested image and 6 mandibular molar image. The segmentation algorithm divides the original image into tooth region and the background region. In order to evaluate the 'true' fraction region of the dental X-ray image, the ground truth is built according to the region that delineated by a doctor. The proposed algorithm is implemented with Matlab software. 


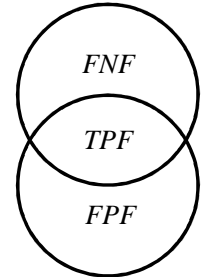

Fig. 6 The corresponding of $T P F, F N F$, and $F P F$

The validation of the csFCM-GK algorithm performance is compared with csFCM algorithm[11]. The parameter $m$ indicates the degree of fuzziness is set by 2, the parameter $r$ and $s$ that influence on the final membership values and cluster center is set by 2 , the windows neighborhood size $(N B)$ is set by $3 \times 3$. Using $m=2, r=2, s=2$ and $N B=3 \times$ 3 provide superior results for csFCM and csFCM-GK algorithms.

The results indicate that the local and global membership values are required involving the process to generate the final membership values and cluster center with the equal importance of membership value $\mu_{i j}$ and membership of spatial information value $u_{i j}$. In dental X-ray image, there is a strong correlation between neighboring pixels. However, windows neighborhood size $N B=3 \times 3$ provides equal results with $5 \times 5$ and $7 \times 7$ but diminishes time running gradually.

Both csFCM and csFCM-GK are processed using flowchart in Fig. 3. After the original dental X-ray image is segmented using those algorithms, the segmented image is changed into the binary image (black for the background and white for the tooth) according to the membership value of the clusters.

The visualizations of dental X-ray image segmentation results are presented in Fig. 5 that illustrates the segmentation of dental X-ray image 1, 2, 6, 8 and 9. The first and fifth row are the mandibular left molar teeth, the second and third row is the mandibular right premolar, and the fourth row is the mandibular right molar. The original image in Fig. 5(a) show the low quality and low contrast of the dental X-ray image in the jpg form. Fig. 5(b) and Fig. 5 (c) present the segmentation result for csFCM and csFCMGK method.

Table 1 presents the segmentation result of csFCM-GK comparing with csFCM algorithms in evaluation criteria. The bold-face type display the best value of $F N F, F P F$ and $T P F$. It can be observed that the dental X-ray image 1, 2, 6, 8 and 9 have better result for $F N F$ and TPF (bold-face) using csFCM-GK than csFCM, in the other hand, csFCM-GK get worse value of $F P F$ than csFCM. The other dental X-ray image shows the same $F N F, F P F$ and $T P F$ values. Therefore, average of $F N F$ and $T P F$ values using csFCMGK are better than csFCM and vise versa for FPF value. According to those evaluations, The proposed csFCM-GK algorithm outperforms for $F N F$ and $T P F$, which it means the teeth region segmented by proposed algorithm is closer to the ground truth as shown in Fig. 5(e). On the other hand, csFCM get smaller FPF value than proposed method. It means the recognizable background region of the proposed method not well enough in allocating it. However, this result does not affect the expected region of interest.

\section{Discussion}

This paper concentrate on the dental X-ray image segmentation with fuzzy clustering approach. Dental X-ray images have a more complex structure than traditional images because of the relationship between various parts and low-quality image due to noise, low contrast, errors on data scanning and etc.

Conditional spatial fuzzy c-means clustering (csFCM) which incorporates a conditioning effect with a condition variable improve robustness to noise and inhomogeneities, thus providing superior segmentation results[11]. The conditional variables $r$ and $s$ describe the level involvement of pixel into membership values and cluster centers. In dental X-ray image, there is a strong correlation between the neighboring pixel and the weighted membership is defined the correlation between global and local information. Therefore the involvement of neighborhood reduces the number of spurious blobs. But in a homogeneous region, the spatial function only reinforces the original membership and resulting in an unchanged clustering. However, windows neighborhood size $N B=3 \times 3$ provides equal results with 5 $\times 5$ and $7 \times 7$ but diminishes time running gradually.

In the proposed algorithm, the Gaussian kernel that added to the objective function displaces the Euclidean distance used in csFCM. Euclidian distance clusters the pixels in the data space. Euclidean distance determines the shortest distance between two-pixel intensity values. However, Euclidian distance has failed to detect outlier data. Gaussian kernel transforms data space into feature space effectively producing better clusters than csFCM as shown in Table 1.

Gaussian radial base function (RBF) that used in this algorithm is simpler and requires inexpensive computation. The variance $\sigma^{2}$, that calculated from the global pixel and neighborhood pixel values without any input, is quite effective for increased noise and inhomogeneities robustness.

The value of Gaussian kernelized is contained in range value of zero to one. Therefore it is consistent with the range of membership values that control FCM role relatively. The Gaussian RBF value is directly used to the objective function of csFCM-GK and gets better results of dental Xray image segmentation.

FNF and TPF value of csFCM-GK in Table 1 obtain a better result than the csFCM or same result. It means that the Gaussian RBF effectively recognizes tooth region as main part of the dental X-ray image. But FPF value of csFCM obtains a better result than csFCM-GK because of the membership value of csFCM-GK spread around the main sections (teeth area) thus the other tooth structures gain to the background region. However, this result does not affect the expected region of interest. The automatic clustering methodology of the csFCM-GK methodology cannot recognize the pulp as part of the tooth due to the similar intensity with the background. In addition, the methodology also cannot resolve superimposed teeth. Therefore, the semiautomatic methodology can be implemented to obtain more accurate segmentation results on dental X-ray images.

In summary, the proposed csFCM-GK algorithm realizes an accurate and automatic segmentation for dental X-ray image. In general, this proposed clustering algorithm can implement into medical diagnostic support. In this case, the 
result of this algorithm will be used by the author for further research in human forensic identification.

\section{CONCLUSIONS}

In this paper, we propose a new segmentation algorithm for dental X-ray image based on spatial information fuzzy cmeans algorithm called csFCM-GK. Local spatial interaction has incorporated among adjacent pixels in the fuzzy membership function. The Gaussian kernel-based has included to the objective function to enhance robustness to inhomogeneities, noise, and other artifacts. This kernel represents the clustering center as a linear function using Gaussian RBF. Hence, the cluster centers lie in data space using simpler and inexpensive computational. Through the experiments in the dental X-ray image, the proposed csFCM-GK algorithm obtains better segmentation accuracy than csFCM.

The results presented in this paper are preliminary, and further process of dental X-ray image segmentation will be used by the author for forensic identification. There are some fails arise if we use an automatic segmentation algorithm as shown in the experiments. For example, the automatic segmentation cannot achieve a good segmentation for superimposing teeth. In the future, we will develop the algorithms in an interactive mode that effectively segment the dental X-ray image.

TABLE I

Segmentation PeRformance (In PERCENT) COMPARISON BETWEEN CSFCM AND CSFCM-GK

\begin{tabular}{|c|c|c|c|c|c|c|}
\hline \multirow{2}{*}{ Dental image \# } & \multicolumn{3}{|c|}{ csFCM } & \multicolumn{3}{c|}{ csFCM-GK } \\
\cline { 2 - 7 } & $\boldsymbol{F N F}$ & $\boldsymbol{F P F}$ & $\boldsymbol{T P F}$ & $\boldsymbol{F N F}$ & $\mathbf{F P F}$ & $\mathbf{9 1 6}$ \\
\hline 1 & 8.67 & $\mathbf{2 1 . 9 1}$ & 91.33 & 22.60 & $\mathbf{9 1 . 8 4}$ \\
\hline 2 & 23.39 & $\mathbf{3 7 . 6 6}$ & 76.61 & $\mathbf{2 2 . 1 2}$ & 39.68 & $\mathbf{7 7 . 8 8}$ \\
\hline 3 & 18.65 & 12.02 & 81.35 & 18.65 & 12.04 & 81.35 \\
\hline 4 & 8.93 & 90.81 & 91.08 & 8.93 & 90.81 & 91.08 \\
\hline 5 & 9.90 & 47.11 & 90.10 & 9.90 & 47.11 & 90.10 \\
\hline 6 & 28.80 & $\mathbf{1 2 . 7 3}$ & 71.20 & $\mathbf{2 4 . 3 8}$ & 14.26 & $\mathbf{7 5 . 6 3}$ \\
\hline 7 & 52.47 & 4.54 & 47.53 & 52.47 & 4.54 & 47.53 \\
\hline 8 & 12.09 & $\mathbf{2 4 . 5 6}$ & 87.91 & $\mathbf{9 . 4 7}$ & 27.14 & $\mathbf{9 0 . 5 3}$ \\
\hline 9 & 13.62 & $\mathbf{4 8 . 6 5}$ & 86.38 & $\mathbf{1 2 . 1 1}$ & 54.14 & $\mathbf{8 7 . 8 9}$ \\
\hline 10 & 3.29 & 56.02 & 96.71 & 3.29 & 56.02 & 96.71 \\
\hline Average & 17.98 & $\mathbf{3 5 . 6 0}$ & 82.02 & $\mathbf{1 6 . 9 5}$ & 36.83 & $\mathbf{8 3 . 0 5}$ \\
\hline
\end{tabular}

\section{ACKNOWLEDGMENT}

The author would like to thank Ministry of Research, Technology and Higher Education, the Republic of Indonesia which support the scholarship of the doctoral program. We also would like to thank to Faculty of Dentistry, Airlangga University, Surabaya, Indonesia who supplies the panoramic data.

\section{REFERENCES}

[1] Said, E. H., Nassar, D. E. M., Fahmy, G., \& Ammar, H. H. (2006) Teeth segmentation in digitized dental X-ray films using mathematical morphology. IEEE transactions on information forensics and security, 1(2), 178-189.

[2] R. C. Gonzales, R. E. Woods, Digital Image Processing, NJ, Upper Saddle River:Prentice-Hall, 2002.

[3] Scott, J. H. (1977). Introduction to dental anatomy. Edinburgh: Churchill Livingstone.

[4] Jufriadif Na`am,Johan Harlan,Sarifuddin Madenda and Eri Prasetio Wibowo,"The Algorithm of Image Edge Detection on Panoramic Dental X-Ray using Multiple Morphological Gradient (mMG) Method," International Journal on Advanced Science, Engineering and Information Technology, vol. 6, no. 6, pp. 1012-1018, 2016.

[5] Zhou, J., \& Abdel-Mottaleb, M. (2005). A content-based system for human identification based on bitewing dental X-ray images. Pattern Recognition, 38(11), 2132-2142.

[6] Nayak, J., Naik, B., \& Behera, H. S. (2015). Fuzzy C-means (FCM) clustering algorithm: a decade review from 2000 to 2014 , Computational intelligence in data mining: Vol. 2 (pp. 133-149). India: Springer.
[7] Bezdek, J. C., Ehrlich, R., \& Full, W. (1984). FCM: The fuzzy c-means clustering algorithm. Computers \& Geosciences, 10(2), 191-203.

[8] Rupika Nilakant,Hema P Menon and Vikram K,"A Survey on Advanced Segmentation Techniques for Brain MRI Image Segmentation," International Journal on Advanced Science, Engineering and Information Technology, vol. 7, no. 4, 2017.

[9] W. Pedrycz, Conditional fuzzy C-means, Pattern Recognit. Lett. 17 (1996) 576 625-631.

[10] Chuang, K. S., Tzeng, H. L., Chen, S., Wu, J., \& Chen, T. J. (2006). Fuzzy c-means clustering with spatial information for image segmentation. computerized medical imaging and graphics, 30(1), 9-15.

[11] Adhikari, S. K., Sing, J. K., Basu, D. K., \& Nasipuri, M. (2015). Conditional spatial fuzzy C-means clustering algorithm for segmentation of MRI images. Applied Soft Computing, 34, 758-769.

[12] Yang, M. S., \& Tsai, H. S. (2008). A Gaussian kernel-based fuzzy cmeans algorithm with a spatial bias correction. Pattern recognition letters, 29(12), 1713-1725.

[13] Gustafson, D.E., Kessel, W.C., 1979. Fuzzy clustering with a fuzzy covariance matrix. In: Proc. IEEE CDC, San Diego, CA, pp. 761-766.

[14] Tzotsos, A., \& Argialas, D. (2008). Support vector machine classification for object-based image analysis. Object-Based Image Analysis, 663-677.

[15] Lawrence, N. (2005). Probabilistic non-linear principal component analysis with Gaussian process latent variable models. Journal of Machine Learning Research, 6(Nov), 1783-1816.

[16] Baudat, G., \& Anouar, F. (2000). Generalized discriminant analysis using a kernel approach. Neural computation, 12(10), 2385-2404.

[17] Zhang, D. Q., \& Chen, S. C. (2003). Clustering incomplete data using kernel-based fuzzy c-means algorithm. Neural processing letters, 18(3), 155-162.

[18] Filippone, M., Camastra, F., Masulli, F., \& Rovetta, S. (2008). A survey of kernel and spectral methods for clustering. Pattern recognition, 41(1), 176-190. 
[19] Chen, S.C., Zhang, D.Q., 2004. Robust image segmentation using FCM with spatial constrains based on new kernel-induced distance measure. IEEE Trans. Systems Man Cybernet. Pt. B 34, 1907-1916.
[20] Wang, W., Li, J., Jiang, Y., Xing, Y., \& Xu, X. (2015, September). An automatic energy-based region growing method for ultrasound image segmentation. In Image Processing (ICIP), 2015 IEEE International Conference on (pp. 1553-1557). IEEE 